\title{
Evaluation of Infrared Missile Warning Algorithm Suites
}

\author{
Steve Hary: John McKeeman. and Dale Van Cleave \\ Wright Laboratories, Avionics Directorate, Information Processing Branch \\ Wright-Patterson AFB, OH 45433-7301
}

\begin{abstract}
Operalion Desert Storm highlighted the effectiveness of infrared (IR) missile warning systems to detect and track hostile missile threats. This. in turn. has sparked renewed interest in the development of IR missite warnitu algorithms and architectures. The Avionics Dirceturate has funded several IR missile warning algorithm studies and the performance of six algorithm suites is presented in this paper. All suites were evalualed against registered data from two bands of mid-wave IR (MIVIR) imatery and are evaluated for their target decetion performance without the aid of tracking algorithms.

The eflectiveness of each algorithm suite to deted point targets was measured in terms of missed detections and false alarms. The preliminary results show that for targets with low signal to noise ratios (S\R), missile warning algorithm suites which use simple spatial and spectral filter actieve the same performance as more computationally complex algorithm suites.
\end{abstract}

\section{Algorithm Suites}

I raditional IR missile warning algorithm suites use three preprocessing stages to extritct excedances from IR image data. I igure 1 shows the data flow between the preprocessing stages. I irst, the data are spatially litlered to increase the signal to clutter ratio of potential targets in the data. Ifter spatial littering, the data are deinterleaved and used as iuput to a spectral filter. The spectral tiller uses the original data and the spatially tittered data from two or more color bands to produce a high signal to clutter ratio image, $S(x, y)$.

The spectrally filtered data is input to a batckground normalizer and thresholder ( $B, \Gamma$ ) where the data is detected and excedances are passed to a target tracker. The list of excedances contains the $x, y$ beation of each exceedance, and any statistics calculated for the exccedance pixel and its hackground. A tratker would use the excediance information to remote false alarms and pats potential tareets to the operator.

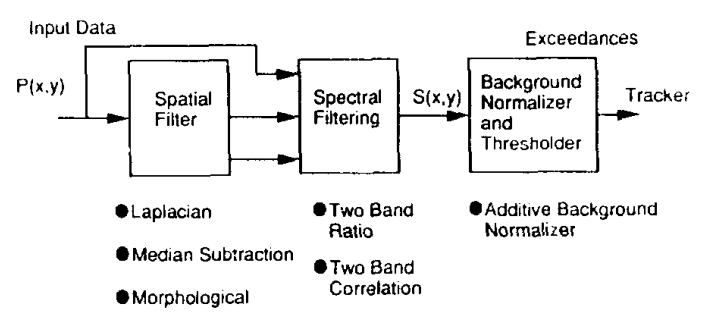

Vigure 1. Data l low for Infrared Misile Warning Suites.

Table 1 shows the six algorithm suites evaluated in this research. Three spatial filters were evaluated: a Laplatian filter, a median subtraction filter (MISI) and a morphedegical filter. Tro spectral filters were evaluated: at two band ratio filler. and a two band corretation filter. Ill algorithm suites used a common atditive bachground normalier and thresholder algorithm.

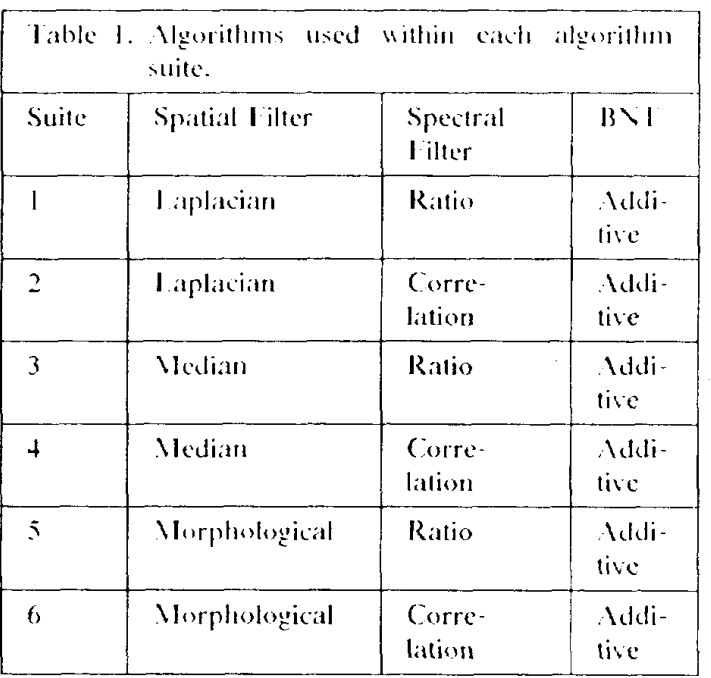

The following subsections of Section 1 discuss the algorithm suites outlined above. Within eath subsection the algorithms used in each filtering stage are detailed. Section 2 discusses the data used to analye the algo rithm suite performance. Section 3 discusses the dita collection method and Section + presents the results and conclusions. 


\subsection{Spatial Filtering}

Spatial filters enhance the siznal to clutter ratio of potential targets in IR imagery through a process known as clutter removal or clutter rejection. Clutter rejection has been the subject of intense research over the past three decades $[1,2,3,4]$. In 1959, Robinson was the first to use nonlinear spatial fiters, known as Rubinson filters, to extract point targets from background clutter in IR imagery [1]. In 1979. Otazo and Parenti showed that targets whose extent is less than one pixel (i.e. point targets) could be eflectively detected using linear, matched, spatial filters [3]. In 1980, Otazo, Tung and Parenti explored spatial fillering algerithms for detecting targels whose extent was slightly greater than one pixel $[+]$.

Takiken et al. developed matched and least mean square (L.MS) spatial filters to increase the signal to noise ratio of targets in a single frame of data [3]. Scribner et al, extended this technique in 1989 to three-dimensions [6]. Their results showed that three-dimensional L.VIS and matched velocity filters perform better than spatial filters, however. these approaches reyuire many banks of velocity filters. In terms of missed detection and false alarm rates. when the target intensity to background noise ratio is low. (i.e $S \backslash R=1$ ) the three-dimensional I. WS filters show a performance improvement by a factor of three over two-dimensional spatial filters. For target intensities to background noise ratios of 3 , the performance of their three-dimensional filters is an order of magnitude better than two dimensional spatial filters The price of this enhanced performance is increased computational complexity and greater memory requirements.

The optimal size and shape of spatial filters used in IR missite warning algorithms is dependent on the noise statistics, the expected intensity distribution, and the $S \backslash R$ of the tareet and backeround clutter. Typically, spatial filters are $3 \times 3,5 \times 5$ or $7 \times 7$ pixels in extent and the operator is typically square. The size and shape of the spatial filter are primary drivers of the litter's computational complexity. For this research. all spatial filters were $3 \times 3$ pixels in extent and the filters were square.

The following subsections discuss the spatial filters used in this rescarch.

\subsubsection{Laplacian Filter}

The laplacian fitter is a standard convolution fitter and the output value, $B(x . y)$, for any input pixel, $P(x, y)$, can be defined as:

$$
\begin{aligned}
B(x, y)=8 & \times P(x, y)-[P(x-1, y-1)+P(x-1, y)+ \\
& P(x-1, y+1)+P(x+1 y-1)+P(x+1 y)+ \\
& P(x+1 y+1)+P(x, y-1)+P(x, y+1)]
\end{aligned}
$$

\subsubsection{Median Subtraction Filter}

Another spatial filter used in WIIIR inagery is the median subtraction filter (IISI) $[7,8]$. Here, the median value of the cight nearest neighbors of the center pixel is subtracted from the value of the center pixel. Havlicek first reported on the performatuce of a two dimensional (eight neighbor) WSI on WIVIR imagery in 1988 [7]. Barnett. in 1989, reported on the statistical propertics of one-dimensional (hree point) MSF's and compared their performance to spatial (nine point) 1.11S filters. Barnett's $\mathrm{MSF}$ provided performance rivaling that of the L.IS filters for targets in mild clutter. however, it performed poorly in scenes containing severe clutter [8]. Birnett's filter required twenty five percent lewer operations per pixel than the I. VIS spatial filter. The eight point VISI proposed by llavlicek required wice the computational complexity of linear spatial filters, but provided better performance than Barnett's MISI. In this research, the llarlicek IISI: was used as the baseline median filter algorithm.

The IISF rank orders the cight nearest neighbors of the input pixel $P(x . y)$ by sorting them from smallest to largest. The ordered pixels are then labeled $x_{n}, x_{1}, x_{2}, \ldots, x$. For an hand of data, the value of the output pixel from the MSF, $B(x . y)$, is defined as:

$B(x, y)=P(x, y)-I f(x, y)$

where $M(x, y)$ is defined as:

$M(x, y)=\frac{1}{2}\left[x_{3}+x_{4}\right]$

The output pixel value is the median value of the eight nearest neighbors of the center pixel subtracted from the value of the center pixel.

\subsubsection{Morphological Filter}

In 1988, Ockman et al. used high-pass. morphological spatial filters to enhance the signal to noise ratio of WIWIR IR Search and Track (IRSI) imagery [9]. These non-linear filters reduce the ringing asseciated with linear spattial filters, but they require more operations than lincar spatial filters.

Morphological filtering uses the structure of the background clutter as part of the clutter removal process. Specifically, a shape operator which approximates the structure of the clutter is convolved with the image. Pixels within the boundaries of the shape operator are used to determine the output. The most commonly used shape operators are a $3 \times 3$ pixel cross operator and a 3 $x 3$ pixel square operatur. The cross operator uses the center pixel and its four nearest neighbors to compute the output. The square operator uses the center pixel and its eight nearest ncighbors 10 compute the output. 
A morphological filter consists of repeated applications of two basic morpholegical filtering operations: dilations and crosions. Figure 2 shows that the morphological filter used in Vlyorithm Suites 5 and 6 consists of four successice filtering passes: dilation, erosion, erosion and dilation, respectively.

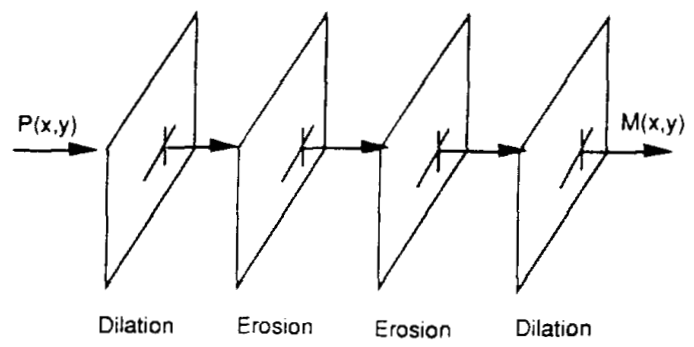

Tigure 2. I low diagram for morphological filter.

As an example. consider a ditation using a square shape operatur. Let the values of the center pixel $\mathrm{P}(x, y)$ and its eight nearest neighbors be defined as: $x_{0} . x_{1}, x_{2}, \ldots, x_{8}$ The value of the output pixel from the dilation, $D(x, y)$ is definted its:

$D(x, y)=\max \left[x_{0}, x_{1}, \ldots, x_{s}\right]$

An crosion replaces the center pixel with the smallest pixel value from the pixels within the boundaries of the shape operator. Consider an erosion using a cross shape operator. Let the values of the enter pixel $P(x, y)$ and its four nearest neighbors be defined as: $x_{0}, x_{1}, x_{2}, \ldots x_{4}$ then, the valle of the output pixel from the crosion. l(x,y) is defined as:

$I(x, y)=\min \left[x_{j}, x_{1}, \ldots, x_{1}\right]$

Iiqure 2 shows that the morptrolugical filter lirst dilates the pixels in the input image, P(x,y). The dilated output image is then croded in two sequential erosion passes. The eroded innage is then ditated in the fometh pass of the morpholegical filter.

Since the morpholonical filter shemen in ligure 2 is a low-pass filler, its linal output is subtrated from the input inage to provide the bigh pass filter used in this research. I or any band of data, the value of the output pixel from the norphological filler, $B(x, y)$, is defined as:
$B(x, y)=P(x, y)-. M(x, y)$

where $\mathrm{I}(x, y)$ is output from the last dilation pass.

\subsection{Spectral Filtering}

Spectral filters further entance the signal to slutter ratio of polential threats in IR imagery by using target specific emissions in two or more color hands. Wlissile and aircraft threat emit laree amounts of radiation in the 4.2-4.8 $4 \mathrm{~m}$ spectrum. Within this band. however, radiation emitted in the $4.4-4.6 \mu \mathrm{m}\left(\mathrm{CO}_{2}\right)$ band is completely absorbed by the atmosphere. Therefore, when discrimi nating hostile missile threats, radiation in the $4.6-4.8$ an band is considered the primary band. A secondary hatud of interest is the $3.85-4.15$ um region. Other bands of interest within the spectral bandwidth (3.0 - 5.0 (a) of an InSt focal plane array (FPA) include guard bands around the primary and secondary bands. In the following discussion. ratiation in the $3.85-4.15 \mathrm{tan}$ region will be called Band 1 , and radiation in the $4.6-4.8 \mathrm{~mm}$ region will be alled Band 2.

Spectral filters assume that energy emitted in Band 2 by missiles is much greater than energy emitted in Band $i$. To discriminate between missiles and clutter. spectral filters also assume that the radiation from backgrouml clutter varies little between bands. Spectral filters use these characteristics to cuhance the signal to dutter ratio of potential missile threats.

Several spectral fillering algorithms have been proposed. The simplest algorithms subtract the energy in the seeondary and guard bands from the energy in the primars: band. An amalog implementation for this spectrat filter thas becn proposed for on fiscal plane processing ssistems and digital implementalions fframe subtration) have been proposed by many sources. Other spectral fitcer algorithms use a $5 \times 5$ pixel window around each pixel in multiple bands $t w$ generate a factor, $x$, which is a esitmate of the encegy ratios or correlation between the backgromuds in the primary and secondary hands. After $x$ has been generated. the center pixel in the seeondary band is multiplied by $x$ and the result is subtracted from the center pixel in the primary band. If the center pixel contains a potential target, the result will be a number much larger than zero. Consersely, for clutier, the output of the spectral filter should be near zero.

The spectral filters used in this research are wo-band spectral filters. Iach filter uses Band 2 as the primary band and Band $I$ as the secondary band. 


\subsubsection{Two Band Ratio Filter}

This filter requires two distinct processes. Iirst. for every pixel in the original image data, the two band ratio filter computes $x$, which is a ratio of the background energy in the primary band (Band 2) to the secondary band (Band I). Whe energy ratio is defined as:

$$
\alpha=\frac{\sum_{m=2 n}^{m} \sum_{m=2}^{n} P_{2}^{2}(x+m \cdot y+n)}{\sum_{2 n}^{m=2} \sum_{-2}^{n=2} P_{1}(x+m \cdot y+n)}
$$

where $P$ z $(x, y)$ is a pixel in the untiltered Band 2 data and $P_{1}(x, y)$ is a pixel in the unfiftered Band $I$ data. lote that the point where $m=n=0$ is $n s t$ included in these calculations.

Once $\alpha$ is calculated. the spectrally filtered value for the pixel. $\mathrm{S}(\mathrm{x}, y)$, is computed as a function of $B_{1}(x, y)$, $B_{2}(x, y)$ and $x$ as shown in equation 7 .

$S(x, y)=B_{2}(x, y)-x \times B_{1}(x, y)$

$B_{1}(x, y)$ and $B_{2}(x, y)$ are the spatially filtered values for Bands 1 and 2. respectively.

\subsubsection{Two Band Correlation Filter}

This spectral filter algorithm uses a $5 \times 5$ pixel window around each pixel in two bands to generate a correlation factor. $x$, which is an estimate of the correlation between the background dala in the primary and secondary bands. After $\alpha$ has been generated, the value of the center pixel in the secondary band, $B_{1}(x, y)$, is multiplied by $x$ and the result is subtracted from the center pixel in the primary band $B_{2}(x, y)$.

The value for alpha is defined as:

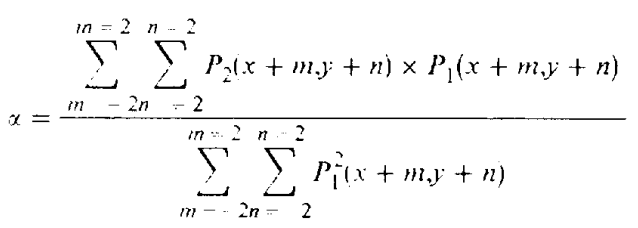

Once $x$ is calculated. the spectrally filtered value for the pixel. $\mathrm{S}(\mathrm{x}, \mathrm{y})$, is computed as a function of $B_{1}(x, y)$, $B_{2}(x, y)$, and $x$ at shown in equation 9.

$S(x, y)=B_{2}(x, y)-x \times B_{1}(x, y)$

where $B_{1}(x, y)$ and $B_{2}(x, y)$ are the spatially filtered values for Bands 1 and 2. respectively.

\subsection{Background Normalizer and Thresholder}

This processing slage detects excecdances in spatially spectrilly filtered data and outputs a list of cxcedances to a tracking algorithm. The most common approach used for target detedion in IR imagery is the contrast box algorithm which is a derivative of constant false alarm rate (CI AR) algorithms used in radar signal processing. Contrast box algorithms assume that the intensity in areas which contain targets will differ significantly from the intensity of their backgrounds.

In 1981, Burton and Benning evaluated four detection algorithms for IR imagery: the contrast hox, the double gated filter, the spoke filter and the superslice algorithm [10]. The contrast box algorithm developed by Texas Instruments demonstrated the best performance.

Figure 3 shows the geometry for contrast box algorithms. Two windows or boxes slide across every pixd in the image. The inner box is matched to the sise of the target and statistics are computed for the pixels within this box. This box is the target box. A second box is used to compute the background statistics around the center pixel and this box is generally much larger than the target box. The mean and standard deviation of the pixels within each box are then computed to crate a contrast metric between the target and its bathground.

In ligure $3, \mu_{i}$ is the mean of the target box. $\mu_{3}$ is the mean of the background box, $\sigma_{\text {: }}$ is the standard deviation of the target box. and $\sigma_{b}$ is the standard deviation of the background box.

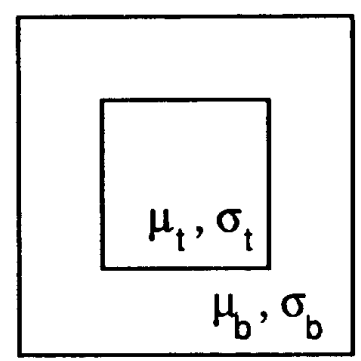

Figure 3. Geometry fior the contrast box algorithm.

The algorithm used in this research is a variant of the contrast box which does not require the computationally intensive tasks of calculating standard deviations. This algorithm assumes that the target box is a single pixel and its walue is compared to the mean energy of its $7 x$ 7 pixel hackground. The resulting contrast metric, $(x, y)$, is defined as:

$C(x, y)=\frac{48 \times S(x, y)}{\sum_{m}^{m=} \sum_{-3 n=-3}^{n=3} S(x+m . y+n)}$ 
where $S(x, y)$ is the value of the spectrally filtered pixel Vote that the term where $m=n=0$ is not included in the hackground mean calculation.

The contrast metric for each pixel is then compared to a threshold, $K$. protided by the user. Pixels whose contrast metric exceeds the threshold are declared excecdances and their intensity values $S(x, y)$ and their average hackeround energy are output to the eracter.

\section{IR Data Generation}

The IR imagery used in the eviluation process consisted of simulated targels embedded in simulated scene data. The background data was created from an IRIV data base and is a sequence of scenes inaged from an aircrafthased missile warning system. Simulated point targets with the spectral characteristics of missile threats were randoml; inserted into the backgrounds.

Seven processes are used to create the realistic IR scene data used to evaluate the candidate IR misisle warning algorithm suites. First, BI.LEMAX II is used to generate a flight path of an aircraft over the earth. The output of BL LCMLX II is a time history of the atrcraft. and hence the IR sensor's location with respect to the ground. This output is used as an input to the Trajectory Inalusis Program (TR-VP) or the Inbanced Surface-to-itr Simulator (ES INIS)

IR:P is used to predict the performance of a launch aircraft, an air launched ground missile and a target in an air-to-air engagenent scenario. TR IP uses the characteristics of the aircraft and the missile, including suid ance and control systen models, to produce an output cortainiag the trajectory for the aireraft and missile rela tive to an earth fixed incrial frame of reference. The output is then converted to a sensor frame of reference.

ISA IIS is similar to TR.YP except that it provides modcling for surface-to-air engagements rather than air to air engagements. The output of CSIIIS is a trajectory file similar to that generated from TR.AP.

The next process in creating the data is calculating the IR signatures of the latunch aircraft and the missile. The launch aircraft IR signatures are derived from the I I I ) signatures database. The missile signatures, howeser, were derived from two sosurces: the I I I) signatures data base and from the Signature of Air-to. Air Mlissiles After Burnout II (SA IMBO II) simulation program. The FID data base provides signatures for missiles during their burn phases, and S M A.MBO II was used to model the post hurnout missile signatures.

To create the background, the target aircraft's trajectory file is imput to IR Background Simulation Computer Program (IRSINIAS). This program creates the IR background as viewed from the target aircratt's sensor by projecting the sensor pixels onto the ground. The eround dala is supplied from the IRIMI Port lleuncme data base. IRSIVIAS inputs the trajectory tile of the target aircraft and outputs a set of hatkground scenes which have been altentated for atmospheric transmission and oplic effects.

The missile and latunch velicle signatures are attenuated for atmospleric and optics effects through the IR I ARCill program. I his program inputs the flight profiles of the target aircraft. the launch aircraft and the missile. The output from this program is a projection of the missile and launch vehicle signatures onto the $\mathrm{P} P$ ?.

The latst data generation step combines the backeround scene with the target scene by adding the pixel irradiances. The program tITD Tl I also adds simmlated electsonic noise to the final image. The linal output is a digitised IR seene of targets embedded in batkgrounds with varsing amounts of moise.

The data set used in this research consisted of 100 sequential frames of regitered WIIIR datit. fach frame represents imagery from a $128 x 128$ pixel InSb IP. that has been imaged at 30 frames per second. The inserted launch aircraft and missile targets were removed from the data set. Then, one hundred point tardets with the same spectral characteristics as misiles in their burn stages were randomly inserted into each ditta frame resulting in 10.1000 total targets. The six algorithm suites were then tested using the wo data sets.

\section{Data Collection Method}

Io measure the effectiveness of each algorithm suite, a false alarm rate versus missed detection rate metric is used. The sirnal to noise rattio for all targets in the data sequence was baried from 1.1 to 2.0 in steps of 0.1 . NII targets were initially inserted with a signal to noive ratio of 1.1 in the primitry band, Band 2 . Ten different values, $K$, were used as the theshold of the bachground usmalizer and thresholder and the number of missed detections and false atarms were recorded for each walue of $K$. Ihe missed detection rate (VIDR) is defined as the number of missed detections divided by the futal number of inserted lareets. The fatse alarm rate (I IR) is defined as the number of false alarms divided by the total number of pixels. The SIDR and I AR recorded by varying $k$ resulted in the data points needed for one performance curse for each iuput signal to noise ratio. ligure 4 . shows atl example family of curses for the performante of Ngorithm Suite $t$. As expected. the performance of Vlgorithm Suite $t$ improves, in terms of fewer missed delections and false alarns, with increasing input signal to moise ratios. 


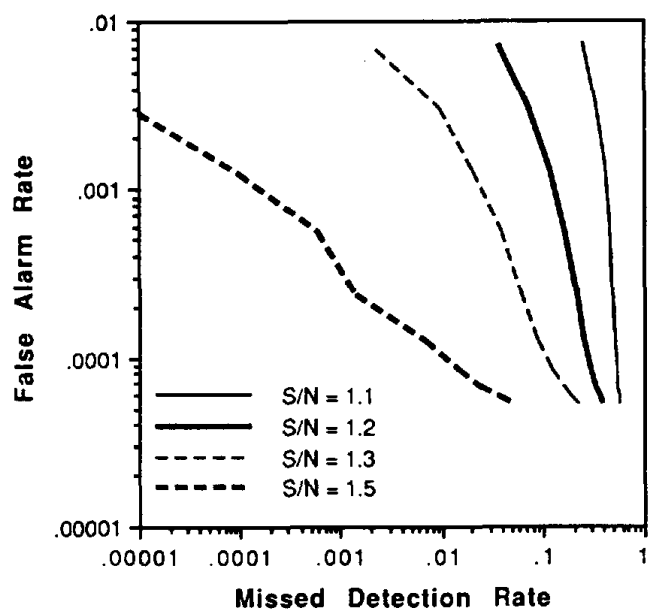

Figure 4. Performance of Algorithm Suite 4.

Performance curves were collected for each algorithm suite and were compiled to make the performance comparison curves presented in Section 4 .

\section{Results and Conclusions}

ligures 5, 6, 7 , and 8 show the performance of each algorithm suite against target with signal to noise ratios of $1.1,1.2 .1 .3$, and 1.5 . respectively. Tahle 2 shows the relatice computational complexity of each algorithm stite in terms of the number of adds. nultiplies, legieal operations and disides for each pixel processed. Sote that the number of additions includes subtractions as well.

figures $5,6,7$, and 8 show that the performance of the algorithm suites varied little based on the spatial fitter used. This result is not unexpected since the spatial filters used in this research were all designed to pass point targets

The results also show that the ratio spectral filter performs as well as the correlation spectral filter when evaltated against registered imagery. The ratio spectral filter requires fewer computations, which may alleviate the processing requirements of IR missile warning sistens. The performance curves show that good detection performance can be achieved against targets with low signal-to-noise ratios by using relatively simple two-dimensional IR missile warning algorithm suites.

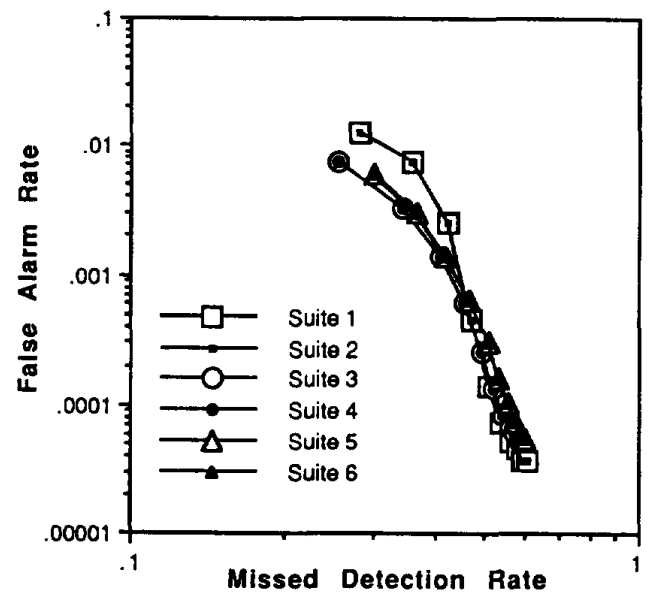

Figure 5. Performance of Algorithm Suites Against 1.1 $S \backslash R$ Targets

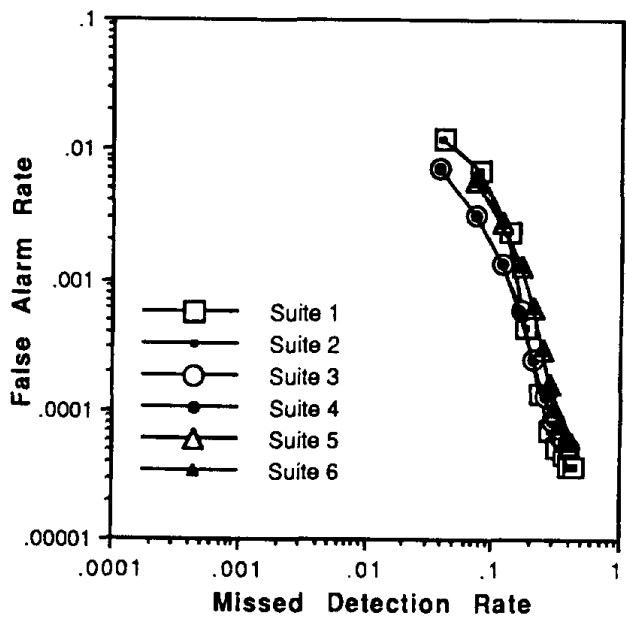

Higure 6. Performance of Algorithm Suites Against 1.2 S $\backslash R$ Tirgets 


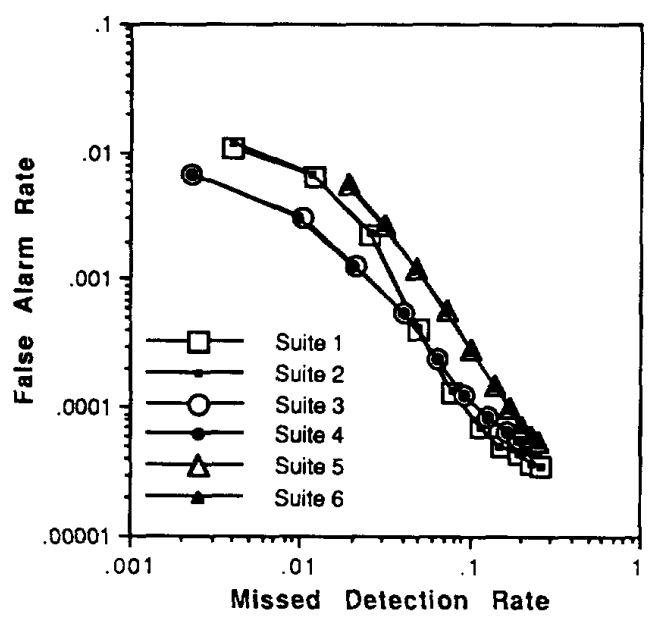

Figure 7. Performance of Algorithm Suites Against 1.3 S. R largets

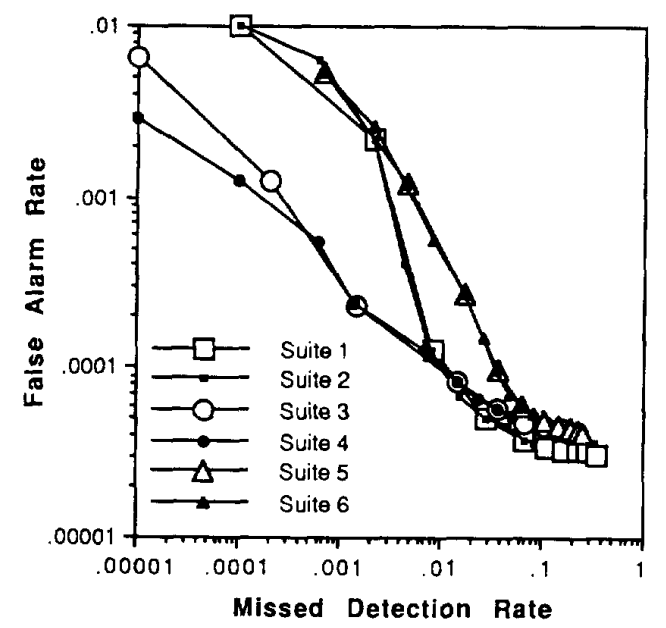

l igure 8 . Performance of Ngorithm Suites Against 1.5 S VR I argets

\begin{tabular}{|l|l|l|l|l|}
\hline \multicolumn{3}{|c|}{ Table 2. Per Pixel Compulational Complexily of } \\
Ngerilhm Suites.
\end{tabular}

\section{References}

Robinson, D., Mellods of Bickgroumd Description and Their Luility, Proceedings for the IRE, Vol. 47. No. 9, 1959.

2

Pratt, W. K., Vector Space Iormulation of Two Dimensional Signal Processing Operations. Com puter lision, Ciraphics and Image Processing, Vol. 4. 1975.

3

Ota\%o, J. J. and Parenti, R. R., Digital Filters fir the Detection of Resolved and Lnresolved largets limbedded in Infrared Scenes, Sl'tr, Vol. 178, 1979.

+ Otazo, J. J., Tung. I. W., and Pareti, R. R., Digital I illers for Infrared larget Acquisition Sensors, SPIE, Vol. 238, 1980, pp. 78-90.

5 Takken et al., least-mean-syuare litter for $\mathbb{R}$ Sensors. Applied Optics, $18(24),+210-4222,19-9$.

6 Scribner. D. A. et al, 3-1) L. MIS Filtering Techniques for Detection of Moving Targets Against Clutter Backgrounds, VRI report, 7 January 1989.

7 Haslicek. J. P.. Median Filtering for Target Detection in an Airberne threat 11 arning System. Waster's Ihesis Virginia Tech. 1988

8 Barnett, J. Statistical Analysis of Median Sub traction I iltering with Application to Point Target Defection in Infrared Backgrounds. SPll, Fol. 1050. pp. 10-18. 1989.

9 Ockman. $x$. J., et al., Application of Morpholngical lilters a Infrared Search and I rack Signal Processing, Martin Marietta, 1988.

10 Burton, 11 . and Bennity, C., Comparison of Infrared Imaging Detection Vlgorithms. SPII Vol. 302. pp. 26-32, 1981

11 Dudani, S. A., Smithgall. B., and Rubins, P.. Target Acquisition and Ixtraction form Cluttered Batckgrsunds, SPll: Vol. 302, pp. 43-50.1989.

12 WeWilliams, J. K.. and Srinath, YI. D., Performance Analysis of a Target Detection Switem Ling Infrared Imagers. Il II Tramsations on Serospice and Flectronic Sistems, Vol. 20, Vo. 1. 1984. pp. $38+48$ 